\title{
Simultaneous Wood Defect and Species Detection with 3D Laser Scanning Scheme
}

\author{
Zhao Peng, Li Yue, and Ning Xiao \\ Information and Computer Engineering College, Northeast Forestry University, Harbin 150040, China \\ Correspondence should be addressed to Zhao Peng; bit_zhao@aliyun.com
}

Received 18 September 2016; Accepted 22 November 2016

Academic Editor: Sulaiman Wadi Harun

Copyright (C) 2016 Zhao Peng et al. This is an open access article distributed under the Creative Commons Attribution License, which permits unrestricted use, distribution, and reproduction in any medium, provided the original work is properly cited.

\begin{abstract}
Wood grading and wood price are mainly connected with the wood defect and wood species. In this paper, a wood defect quantitative detection scheme and a wood species qualitative identification scheme are proposed simultaneously based on 3D laser scanning point cloud. First, an Artec 3D scanner is used to scan the wood surface to get the 3D point cloud. Each 3D point contains its $X, Y$, and $Z$ coordinate and its RGB color information. After preprocessing, the $Z$ coordinate value of current point is compared with the set threshold to judge whether it is a defect point (i.e., cavity, worm tunnel, and crack). Second, a deep preferred search algorithm is used to segment the retained defect points marked with different colors. The integration algorithm is used to calculate the surface area and volume of every defect. Finally, wood species identification is performed with the wood surface's color information. The color moments of scanned points are used for classification, but the defect points are not used. Experiments indicate that our scheme can accurately measure the surface areas and volumes of cavity, worm tunnel, and crack on wood surface with measurement error less than $5 \%$ and it can also reach a wood species recognition accuracy of $95 \%$.
\end{abstract}

\section{Introduction}

Wood species and wood defects are two key issues in the wood quality assessment so as to judge the physical property and commercial value of different wood products (e.g., wood veneer, lumber, or board) correctly [1]. Some visual image characteristics have been used in the wood species recognition and can be divided into two general categories: wood surface's texture analysis $[2,3]$ and its color analysis $[4,5]$. Recently, the wood spectral reflectance characteristics are also exploited for the species classification. The more common schemes in the literature consider the vibration spectroscopy [6,7] and the Raman spectroscopy [8]. For example, Piuri and Scotti present a scheme for the wood species classification based on the analysis of fluorescence spectra [9].

As for the wood surface's defect detection, the spectral analysis and laser scanning schemes are usually used to fulfill the qualitative detection on the wood external defects (e.g., cavity, worm tunnel, knots, or erosion) [10-12]. Researchers also have proposed some schemes for detecting wood internal defects by using X-rays, gamma rays, microwaves, and longitudinal stress waves [13-15]. For example, Wang et al. combine the wavelet transform and neural networks to analyze and recognize different types and sizes of wood internal defects using an ultrasonic device [16].

However, simultaneous investigations on wood species and wood defect detections are scarcely performed to make an objective wood quality assessment. In fact, wood species detection and wood defect detection are usually performed with different instruments or technologies. Even if the same instrument or technology such as spectral analysis may be used for wood species and defect detection, the mutual disturbance exists so that the detection accuracy is low. For example, the wood species recognition accuracy is not good for wood veneer if there are many defects on wood surface by using spectral reflectance features. To solve this hard issue, we use a 3D laser scanning instrument to make a qualitative wood species recognition and a quantitative wood defect measurement (i.e., the precise measurement of surface area and volume of every external defect such as cavity, worm tunnel, and crack on the wood product's surface) simultaneously. Therefore, the wood detection efficacy is improved by using our scheme and it can provide a better basis for the subsequent wood quality assessment. 


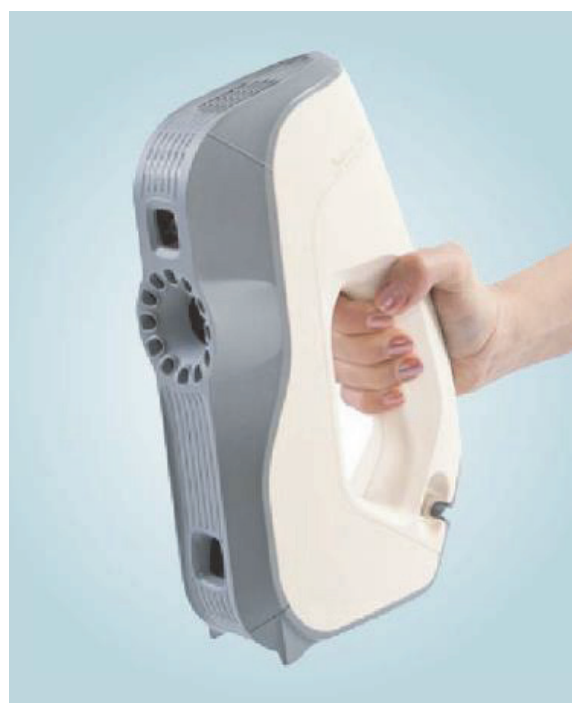

Figure 1: A portable Artec 3D laser scanner.

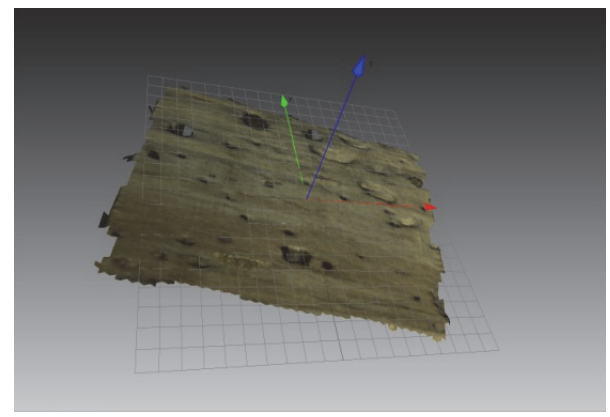

FIgURE 2: Scanned wood surface in the Artec Studio 9.

\section{Materials and Methods}

2.1. $3 D$ Scan. The $3 \mathrm{D}$ scan is a nondestructive detection technology based on laser processing with a fast scan speed and a high scan accuracy. Therefore, it is usually used in the object's $3 \mathrm{D}$ reconstruction. In our scheme, a portable Artec 3D laser scanner is used to get the wood surface's $3 \mathrm{D}$ point cloud, as illustrated in Figure 1. This scanner is small and portable with an adjustable flash lamp and with a $3 \mathrm{D}$ scan resolution of $0.5 \mathrm{~mm}$. It is used conveniently with a number of data storage formats. This scanner is equipped with software system Artec Studio 9 to read the scanner data and then display the scanned object's surface. The object's surface is calibrated with the instrument's $X O Y$ plane automatically by this software and then the object's surface is stored as a file with OBJ format. For example, Figure 2 illustrates the scanned wood surface in the Artec Studio 9. Each scanned point contains its $X, Y$, and $Z$ coordinate and its RGB color information. The point's $X$, $Y$, and $Z$ coordinates are used in the defect detection, while its RGB information is used in the species recognition.

2.2. Defect Segmentation. In this section, two steps need to be executed to segment different defects. First, every point's $Z$ coordinate is compared with the set threshold to determine whether this point is a depressed defect (i.e., cavity, worm tunnel, and crack) point. Second, the retained defect points are processed with a deep preferred search algorithm to segment different defects. The defect points which belong to the same defect will be marked with the same number and displayed with the same color. A detailed flow graph is illustrated in Figure 3, and different defects are displayed with different colors, as illustrated in Figure 4.

2.3. Defect Measurement. For every defect such as every cavity or worm tunnel, we propose an integration scheme to calculate its surface area and volume. To achieve this goal, we need to extend each defect point into a small surface. As illustrated in Figure 5, every three adjacent defect points form a triangle plane, while each defect point is the vertex of six triangle planes. Therefore, we should extend each defect point into a regular hexagon surface model, as illustrated in Figure 6. In Figure 6, the point $O$ is the current processed defect point with six adjacent points $A \sim F$. We draw six midnormals on $O A, O B, \ldots, O F$, and these six midnormals intersect each other at the six vertices $P, Q, R, S, N$, and $T$. These six cross-points form a small purple regular hexagon. In fact, we finally extend each defect point into this small purple regular hexagon $P Q R S N T$.

As for the surface area computation of every defect, we should firstly calculate the area of the small purple regular hexagon $P Q R S N T$. Define $L$ as the mean distance value of parallel opposing sides such as $P Q$ and $S N$ in $P Q R S N T$, and this distance $L$ can be approximated as the mean length value of six sides $O A, O B, O C, \ldots, O F$. After we calculate every defect point's surface area (i.e., the area of $P Q R S N T$ ), then we can summarize all defect point's areas of one defect to get one defect's total surface area, as illustrated in

$$
S=\sum_{i=1}^{n} \frac{\sqrt{3}}{2} \cdot L_{i}^{2}
$$

As for the volume computation of every defect, we should also similarly extend a defect point $O$ into regular hexagon $P Q R S N T$. In this way, we can summarize all defect point's volumes of one defect to get one defect's total volume, as illustrated in

$$
V=\sum_{i=1}^{n}\left(S_{i} \cdot z_{i}\right)=\sum_{i=1}^{n}\left(\frac{\sqrt{3}}{2} \cdot L_{i}^{2} \cdot z_{i}\right) .
$$

In (2), the corresponding volume for every defect point $O$ is approximated as the volume of the regular hexagonal prism, as illustrated in Figure 7. The height $h$ is approximated as $z_{i}$, which is the absolute value of $Z$ coordinate of the $i$ th defect point.

2.4. Species Identification. Wood species identification is performed with wood surface's color information. Each scanned point contains its $X, Y$, and $Z$ coordinate and its RGB color information. The point's RGB color information is used here to calculate the color moment features for subsequent species identification. Obviously the wood surface's defect points will disturb the precise calculations of color moment features and these defect points should be deleted. Fortunately, in 


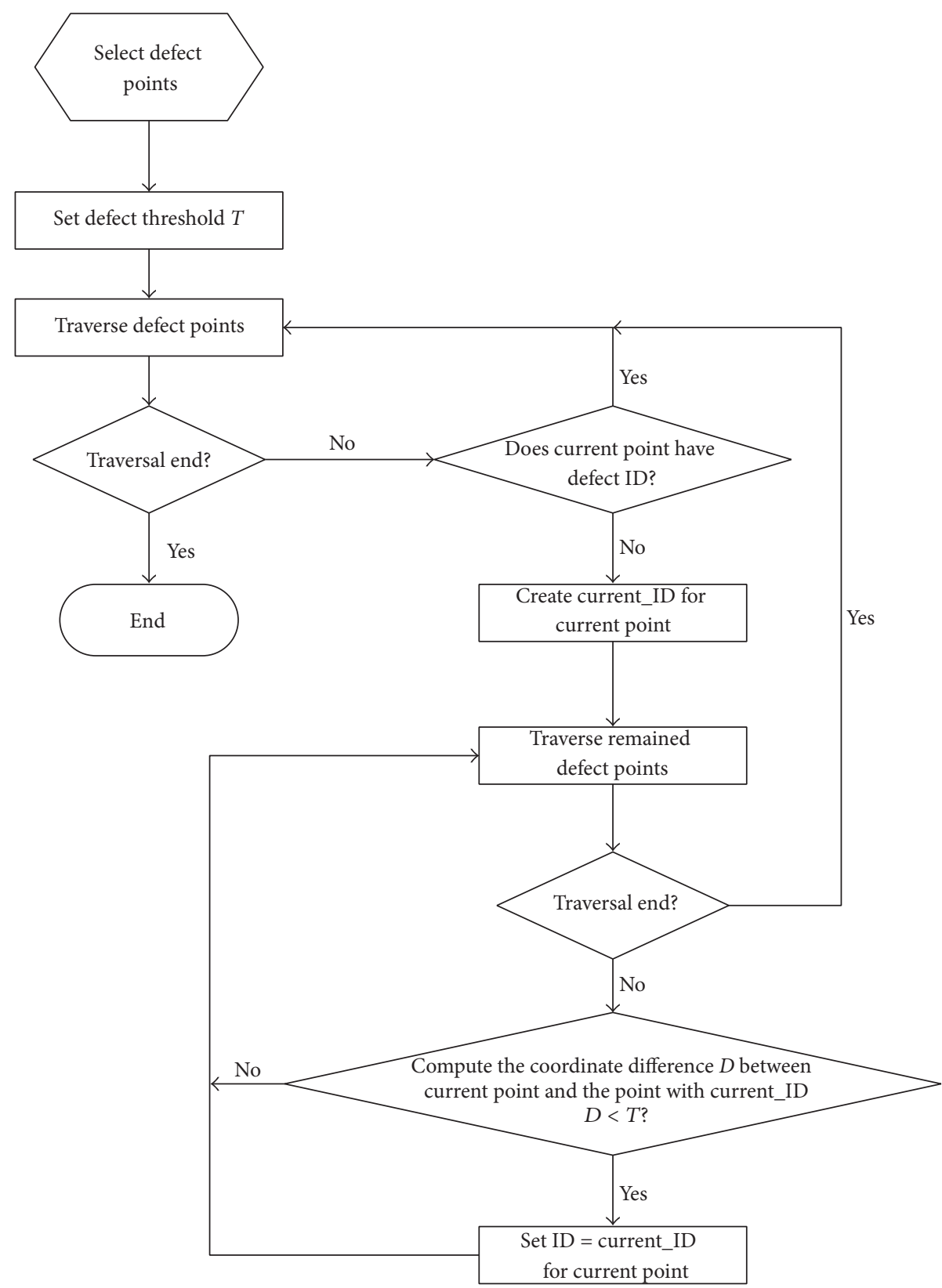

FIGURE 3: The flow graph on wood defect segmentation.

Section 2.2, we have successfully extracted these defect points and therefore the disturbance from defect points can be overcome effectively.

The following three color moments are used for RGB three channels, and we can get 9 classification features. As for the classifier design, the BP neural network is used.

$$
\begin{aligned}
& \alpha=\frac{1}{n} \sum_{i=1}^{n} P_{i}, \\
& \beta=\left(\frac{1}{n} \sum_{i=1}^{n}\left(P_{i}-\alpha\right)^{2}\right)^{1 / 2}, \\
& \gamma=\left(\frac{1}{n} \sum_{i=1}^{n}\left(P_{i}-\alpha\right)^{3}\right)^{1 / 3} .
\end{aligned}
$$

\section{Results and Discussions}

3.1. Defect Detection. We select many wood products which have cavity, worm tunnel, or crack for experiments (one example is illustrated in Figure 8). The Artec 3D scanner is used to get the wood surface's point cloud data, and subsequent defect segmentation and measurement are performed by use of $\mathrm{C}$ language programming. One detailed defect detection result is illustrated in Figure 9.

In order to evaluate our defect detection scheme's measurement accuracy objectively, a simulation experiment is designed here. We select some standard wood boards without any defects on their smooth surfaces and then we use electric drills to drill some pores with different sizes on board surfaces. These drilled pores are the standard cylinder pores 


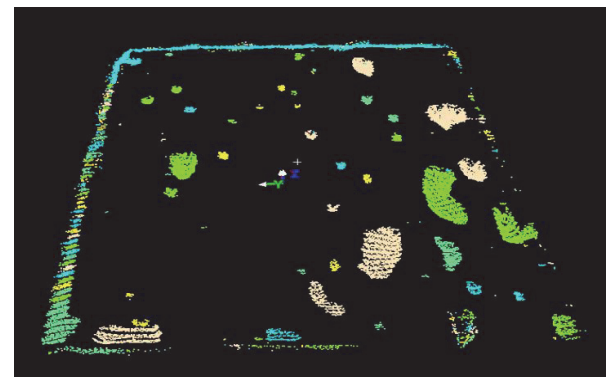

FIGURE 4: The segmented defects displayed with different colors.

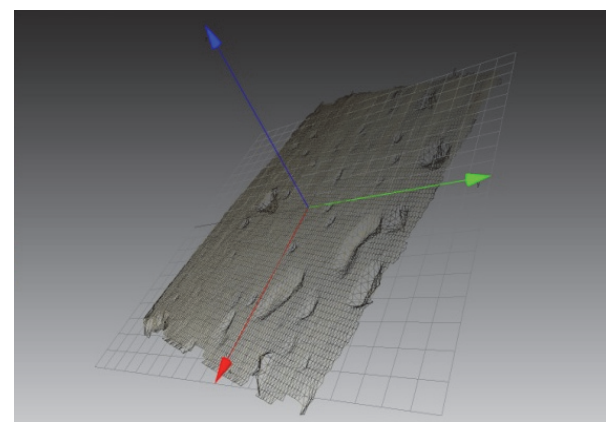

FIgURE 5: Wood surface's grid graph in the Artec Studio 9 without color information.

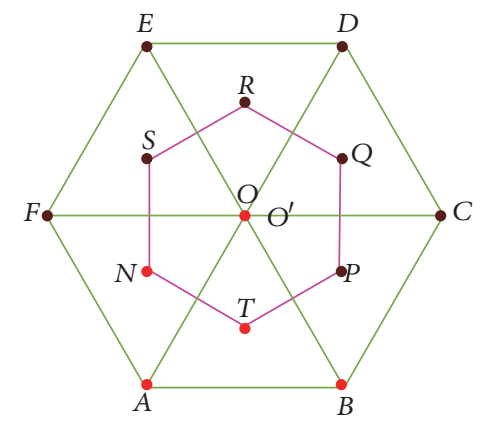

FIGURE 6: A defect point is extended into a purple regular hexagon PQRSNT.

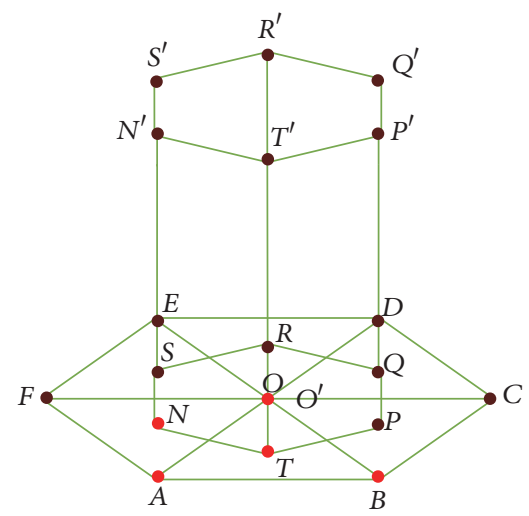

FIGURE 7: The regular hexagonal prism model for volume calculation.

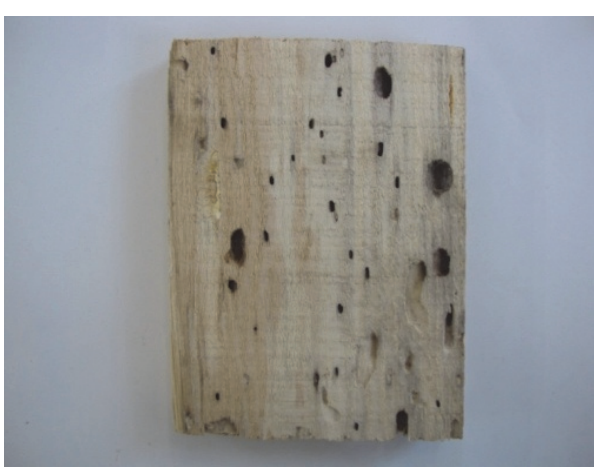

FIGURE 8: The detected wood specimen.

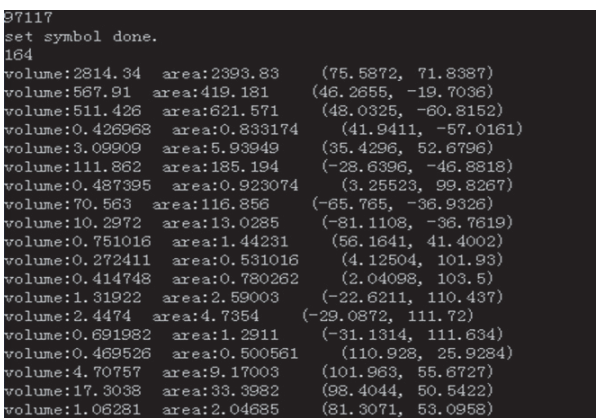

Figure 9: The detailed wood defect's measurement results.

with diameter $4 \mathrm{~mm} \sim 20 \mathrm{~mm}$ and height $1 \mathrm{~mm} \sim 5 \mathrm{~mm}$. We use our scheme to calculate the surface areas and volumes of these cylinder pores and the standard surface areas and volumes are calculated with cylinder's area and volume formulas. By this way, we can objectively determine our scheme's measurement errors and detailed errors are illustrated in Tables 1 and 2 . By comparisons, we can see that our measurement accuracy decreases gradually when the cylinder's height increases. The main measurement error is from the scanning error of Artec scanner, which performs the surface scanning and its scanning error will increase when the depressed defect's height increases. But within the cylinder defect's height $3 \mathrm{~mm}$, our scheme's measurement is good with the relative errors less than $5 \%$.

3.2. Species Recognition. In this section, we perform the wood species identification experiments on five wood species including Betula platyphylla, Populus davidiana, Pinus sylvestris, Picea jezoensis, and Larix gmelinii (i.e., BP, $P D, P S, P J$, and $L G)$ in northeast region of China with 2000 specimens for each wood species (1000 specimens are used as training dataset and another 1000 specimens are used as test dataset). The Matlab 6.5 programming tool is used here and its neural network toolbox is used for BP network's training and identification. The training frequency is 4000 for BP network and its node number is 19 in its hidden layer. The network's training function is trainbr. For each wood species, radial section (RS) and tangential section (TS) are performed, respectively. The detailed results are illustrated 
TABLE 1: Volume measurement error for cylinder cavity.

\begin{tabular}{|c|c|c|c|c|c|c|c|}
\hline Relative error (\%) & $D=4 \mathrm{~mm}$ & $D=6 \mathrm{~mm}$ & $D=8 \mathrm{~mm}$ & $D=10 \mathrm{~mm}$ & $D=13 \mathrm{~mm}$ & $D=16 \mathrm{~mm}$ & $D=19 \mathrm{~mm}$ \\
\hline$H=1.0 \mathrm{~mm}(\%)$ & 3.0 & 3.0 & 3.0 & 3.1 & 3.0 & 3.0 & 3.0 \\
\hline$H=1.5 \mathrm{~mm}(\%)$ & 3.2 & 3.2 & 3.2 & 3.2 & 3.2 & 3.2 & 3.2 \\
\hline$H=2.0 \mathrm{~mm}(\%)$ & 3.5 & 3.5 & 3.5 & 3.5 & 3.5 & 3.4 & 3.5 \\
\hline$H=2.5 \mathrm{~mm}(\%)$ & 3.9 & 3.9 & 3.9 & 3.9 & 4.0 & 3.8 & 3.8 \\
\hline$H=3.0 \mathrm{~mm}(\%)$ & 4.8 & 5.0 & 4.8 & 4.8 & 4.8 & 4.9 & 5.0 \\
\hline
\end{tabular}

TABLE 2: Surface area measurement error for cylinder cavity.

\begin{tabular}{|c|c|c|c|c|c|c|c|}
\hline Relative error (\%) & $D=4 \mathrm{~mm}$ & $D=6 \mathrm{~mm}$ & $D=8 \mathrm{~mm}$ & $D=10 \mathrm{~mm}$ & $D=13 \mathrm{~mm}$ & $D=16 \mathrm{~mm}$ & $D=19 \mathrm{~mm}$ \\
\hline$H=1.0 \mathrm{~mm}(\%)$ & 2.7 & 2.9 & 2.7 & 2.7 & 2.7 & 2.7 & 2.7 \\
\hline$H=1.5 \mathrm{~mm}(\%)$ & 2.9 & 2.9 & 2.9 & 2.9 & 2.9 & 2.8 & 2.9 \\
\hline$H=2.0 \mathrm{~mm}(\%)$ & 3.3 & 3.5 & 3.3 & 3.3 & 3.6 & 3.3 & 3.3 \\
\hline$H=2.5 \mathrm{~mm}(\%)$ & 3.8 & 3.8 & 3.8 & 3.8 & 3.7 & 3.7 & 3.7 \\
\hline$H=3.0 \mathrm{~mm}(\%)$ & 4.5 & 4.5 & 4.7 & 4.8 & 4.8 & 4.9 & 5.0 \\
\hline
\end{tabular}

TABLE 3: Pattern recognition accuracy comparisons for our scheme and ordinary color moment-based scheme (RS).

\begin{tabular}{lccccc}
\hline Scheme & $B P$ & $P D$ & $P S$ & $P J$ & $L G$ \\
\hline Our scheme & $95 \%$ & $95 \%$ & $96 \%$ & $97 \%$ & $96 \%$ \\
Ordinary scheme & $87 \%$ & $85 \%$ & $79 \%$ & $89 \%$ & $89 \%$ \\
\hline
\end{tabular}

TABle 4: Pattern recognition accuracy comparisons for our scheme and ordinary color moment-based scheme (TS).

\begin{tabular}{lccccc}
\hline Scheme & $B P$ & $P D$ & $P S$ & $P J$ & $L G$ \\
\hline Our scheme & $97 \%$ & $95 \%$ & $95 \%$ & $94 \%$ & $94 \%$ \\
Ordinary scheme & $88 \%$ & $88 \%$ & $81 \%$ & $87 \%$ & $85 \%$ \\
\hline
\end{tabular}

in Tables 3 and 4, where we can see that the recognition accuracy of our scheme with defect points deleted is better than that of ordinary color moment-based scheme.

\section{Conclusions}

In this paper, we have proposed a simultaneous wood defect and wood species detection scheme based on 3D scanning and signal processing. It can measure the wood surface's depressed defects quantitatively in terms of area and volume. Moreover, we also find that wood defects usually disturb the wood species identification. Therefore, based on the wood defect's detection results, an improved wood species identification based on color moments is also proposed by deleting those defect points.

For example, the measurement errors for wood defect's areas and volumes can be approximately less than $5 \%$ if the defect's height is less than $3.0 \mathrm{~mm}$. Moreover, the improved wood species recognition scheme can achieve a recognition accuracy of $95 \%$ approximately for 5 wood species. Therefore, our wood quality detection scheme is portable and efficient which can be applied in the practical wood processing factory. In fact, our scheme may be further used in other detection fields such as the surface detections of online industrial products.

\section{Competing Interests}

Dr. Zhao Peng declares that there is no conflict of interests regarding the publication of this paper.

\section{Acknowledgments}

This research is supported by the National Natural Science Foundation of China with Grant no. 31670717 and Heilongjiang Province Natural Science Foundation with Grant no. C2016011. It is also supported by the New Century Excellent Talents in University with Grant no. NCET-120809 and the Fundamental Research Funds for the Central University with Grant no. 2572014EB05-01.

\section{References}

[1] A. K. Moore and N. L. Owen, "Infrared spectroscopic studies of solid wood," Applied Spectroscopy Reviews, vol. 36, no. 1, pp. 65-86, 2001.

[2] M. S. Packianather and P. R. Drake, "Neural networks for classifying images of wood veneer. Part 2," International Journal of Advanced Manufacturing Technology, vol. 16, no. 6, pp. 424433, 2000.

[3] M. Nakamura, M. Masuda, and K. Shinohara, "Multiresolutional image analysis of wood and other materials," Journal of Wood Science, vol. 45, no. 1, pp. 10-18, 1999.

[4] Q. Wei, Y. H. Chui, B. Leblon, and S. Y. Zhang, "Identification of selected internal wood characteristics in computed tomography images of black spruce: a comparison study," Journal of Wood Science, vol. 55, no. 3, pp. 175-180, 2009.

[5] J. E. Phelps and E. A. Mcginnes, "Growth-quality evaluation of black walnut wood, part 2-color analysis of veneer produced on different sites," Wood and Fiber Science, vol. 15, no. 2, pp. 177185, 1983.

[6] S. Tsuchikawa, Y. Hirashima, Y. Sasaki, and K. Ando, "Nearinfrared spectroscopic study of the physical and mechanical 
properties of wood with meso- and micro-scale anatomical observation," Applied Spectroscopy, vol. 59, no. 1, pp. 86-93, 2005.

[7] C. R. Orton, D. Y. Parkinson, P. D. Evans, and N. L. Owen, "Fourier transform infrared studies of heterogeneity, photodegradation, and lignin/hemicellulose ratios within hardwoods and softwoods," Applied Spectroscopy, vol. 58, no. 11, pp. 1265-1271, 2004.

[8] B. K. Lavine, C. E. Davidson, A. J. Moores, and P. R. Griffiths, "Raman spectroscopy and genetic algorithms for the classification of wood types," Applied Spectroscopy, vol. 55, no. 8, pp. 960966, 2001.

[9] V. Piuri and F. Scotti, "Design of an automatic wood types classification system by using fluorescence spectra," IEEE Transactions on Systems, Man, and Cybernetics Part C: Applications and Reviews, vol. 40, no. 3, pp. 358-366, 2010.

[10] P. K. Lebow, C. C. Brunner, A. G. Maristany, and D. A. Butler, "Classification of wood surface features by spectral reflectance," Wood and Fiber Science, vol. 28, no. 1, pp. 74-90, 1996.

[11] D. E. Kline, C. Surak, and P. A. Araman, "Automated hardwood lumber grading utilizing a multiple sensor machine vision technology," Computers and Electronics in Agriculture, vol. 41, no. 1-3, pp. 139-155, 2003.

[12] J. W. Funck, Y. Zhong, D. A. Butler, C. C. Brunner, and J. B. Forrer, "Image segmentation algorithms applied to wood defect detection," Computers and Electronics in Agriculture, vol. 41, no. 1-3, pp. 157-179, 2003.

[13] F. Longuetaud, F. Mothe, B. Kerautret et al., "Automatic knot detection and measurements from X-ray CT images of wood: a review and validation of an improved algorithm on softwood samples," Computers and Electronics in Agriculture, vol. 85, no. 2, pp. 77-89, 2012.

[14] S. Gao, N. Wang, L. Wang, and J. Han, "Application of an ultrasonic wave propagation field in the quantitative identification of cavity defect of log disc," Computers and Electronics in Agriculture, vol. 108, no. 3, pp. 123-129, 2014.

[15] V. Bucur, "Ultrasonic techniques for nondestructive testing of standing trees," Ultrasonics, vol. 43, no. 4, pp. 237-239, 2005.

[16] L. Wang, L. Li, W. Qi, and H. Yang, "Pattern recognition and size determination of internal wood defects based on wavelet neural networks," Computers and Electronics in Agriculture, vol. 69, no. 2, pp. 142-148, 2009. 

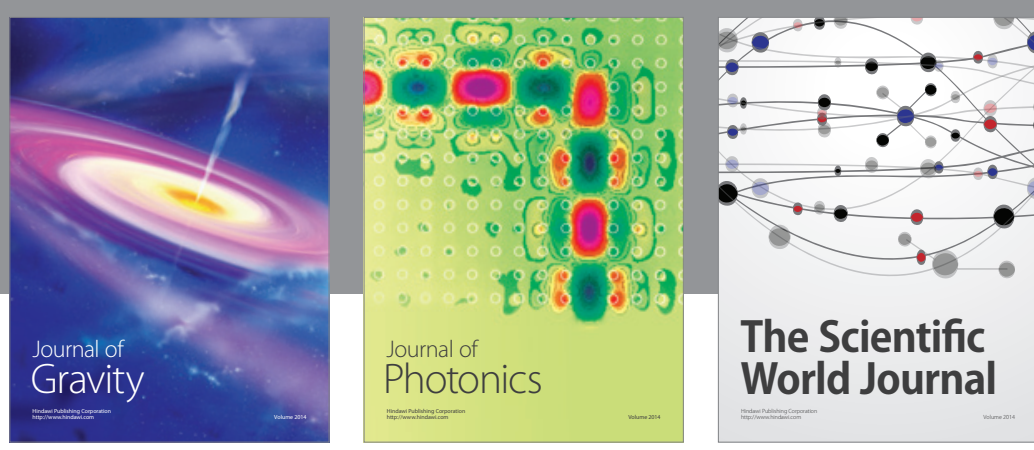

The Scientific World Journal
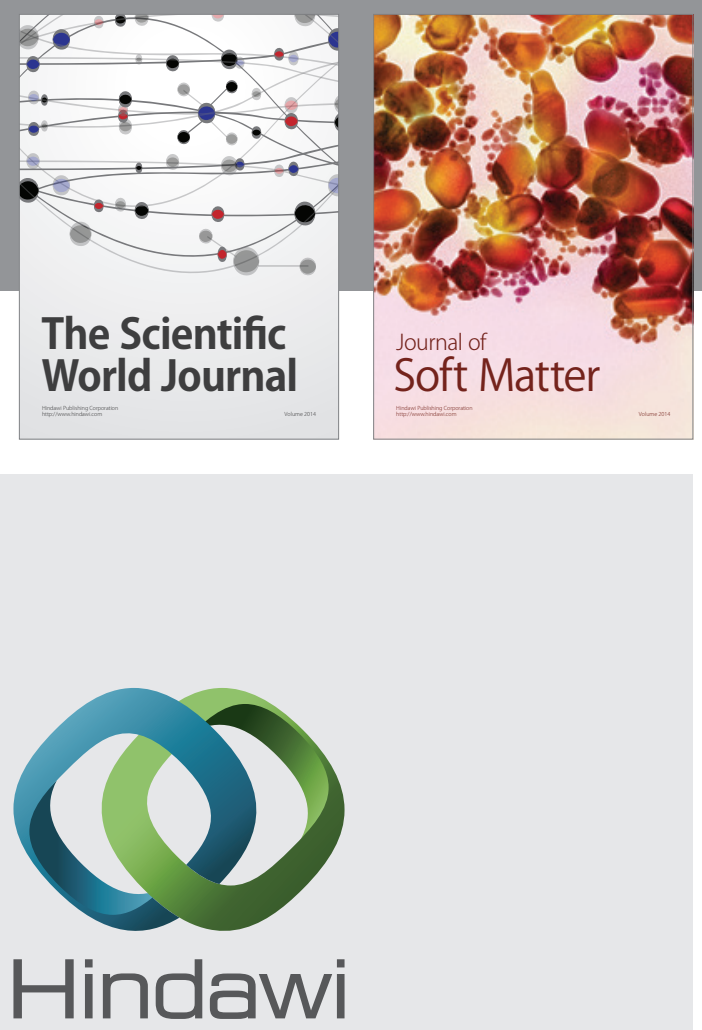

Submit your manuscripts at

http://www.hindawi.com

nternational Journal of

Statistical Mechanics
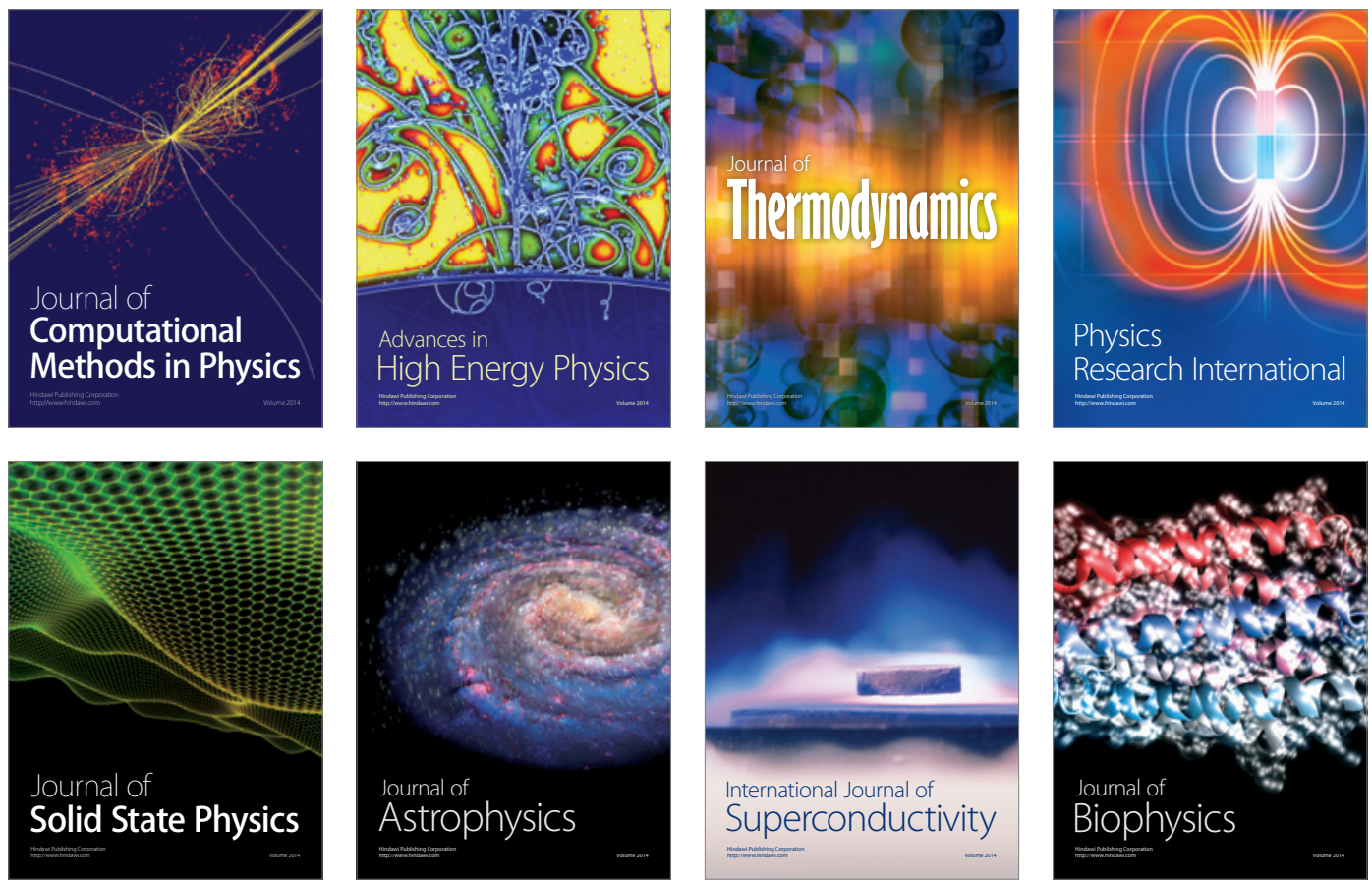
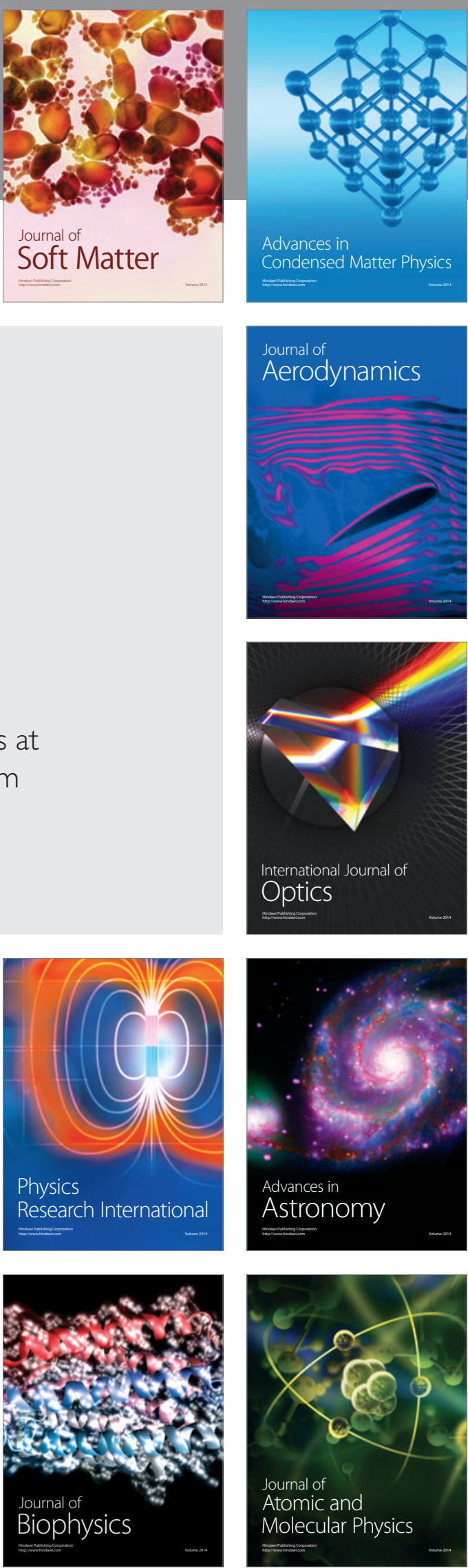